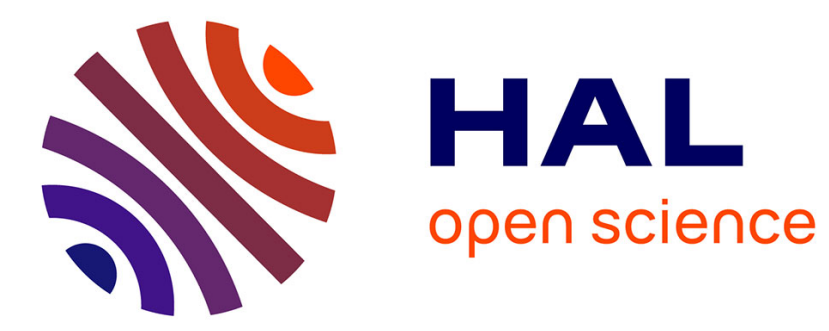

\title{
Targeting the tumor microenvironment in cholangiocarcinoma: implications for therapy
}

Corentin Louis, Julien Edeline, Cédric Coulouarn

\section{To cite this version:}

Corentin Louis, Julien Edeline, Cédric Coulouarn. Targeting the tumor microenvironment in cholangiocarcinoma: implications for therapy. Expert Opinion on Therapeutic Targets, 2021, 25 (2), pp.153162. 10.1080/14728222.2021.1882998 . hal-03129158

\section{HAL Id: hal-03129158 \\ https://hal.science/hal-03129158}

Submitted on 7 Apr 2021

HAL is a multi-disciplinary open access archive for the deposit and dissemination of scientific research documents, whether they are published or not. The documents may come from teaching and research institutions in France or abroad, or from public or private research centers.
L'archive ouverte pluridisciplinaire HAL, est destinée au dépôt et à la diffusion de documents scientifiques de niveau recherche, publiés ou non, émanant des établissements d'enseignement et de recherche français ou étrangers, des laboratoires publics ou privés. 
Targeting the Tumor Microenvironment in Cholangiocarcinoma: Implications for Therapy

Corentin Louis ${ }^{a}$, Julien Edeline ${ }^{\mathrm{a}}$ and Cédric Coulouarn ${ }^{\mathrm{a}}$

a Inserm, Univ Rennes 1, COSS (Chemistry, Oncogenesis Stress Signaling), UMR_S 1242, Centre de Lutte contre le Cancer Eugène Marquis, Rennes, France

ORCID

Corentin Louis https://orcid.org/0000-0002-5513-4394

Julien Edeline $\quad$ https://orcid.org/0000-0002-8289-7741

Cédric Coulouarn https://orcid.org/0000-0002-5692-9586

\section{Contact}

Cédric COULOUARN

Inserm UMR_S 1242, "Chemistry, Oncogenesis, stress, Signaling" (COSS)

Centre de Lutte contre le Cancer Eugène Marquis,

Rue de la Bataille Flandres Dunkerque, Bat D, F-35042 Rennes, France.

E-mail: cedric.coulouarn@inserm.fr 


\section{ABSTRACT}

Introduction: Cholangiocarcinoma (CCA) is a rare but deadly cancer worldwide, mainly as a result of a late diagnosis and limited effective treatments. Targeting the tumor microenvironment (TME) emerged as a relevant strategy in CCA which is characterized by an abundant desmoplastic stroma.

Areas covered: In this review, we describe the role of different compartments of the TME in CCA, focusing on the preclinical rationale for the development of innovative therapies. Relevant literature was identified by a PubMed search covering the last decade (2010-2020).

Expert opinion: Low efficacy of surgery and cytotoxic chemotherapy for a long term cure of patients with CCA emphasize the need for developing innovative therapeutic strategies and companion biomarkers. Single-cell RNA sequencing of the stroma is providing a critical functional characterization of TME in CCA and is paving the way for immunotherapies, as well as cancer-associated fibroblast and extracellular matrixoriented treatments. We believe that the development of treatments targeting different components the TME will produce the best results if thought as a combination with cytotoxic chemotherapy. Positive results would also be clearer if biomarkers are developed to define the population of interest for each combination strategy.

Keywords: Angiogenesis; cancer-associated fibroblast; cholangiocarcinoma; combined therapy; extracellular matrix; immunotherapy; stroma; therapeutic targets; transcriptomics; tumor microenvironment. 


\section{Article highlights:}

- CCA is a deadly cancer worldwide with increased incidence and limited effective therapeutic options.

- Targeting the TME emerges as a relevant strategy in CCA, which is characterized by a dense desmoplastic stroma.

- Gene expression profiling of the stroma provides information on the role of TME in CCA onset and progression and paves the way to design effective immunotherapies.

- Targeting genetic alterations of cancer cells together with TME represents a promising strategy to treat CCA and to overcome drug resistance.

- Targeting the TME should be conducted in combination with cytotoxic chemotherapy with opportunities of synergies. Relevant biomarkers are needed to define the population of interest for each combination strategy.

\section{Introduction}

Cholangiocarcinoma (CCA) is a deadly cancer worldwide with increased incidence and limited effective therapeutic options. CCA comprise different types of solid tumors arising along the biliary tree. Based on the anatomic location, intrahepatic (iCCA), perihilar $(p C C A)$ and distal CCA (dCCA) are distinguished (Figure 1) $[1,2]$. The incidence of CCA is currently increasing in Western countries [1,3]. Treatment of CCA presents numerous challenges, from basic understanding of CCA heterogeneity to clinical aspects related to the silent nature of the disease [1]. Surgery remains the treatment of reference for curative intent. However, a majority of patients could not have their tumor removed as a 
result of locally advanced or metastatic disease at the time of diagnosis [4]. Adjuvant chemotherapy was proven effective, although the benefit was modest and the majority of patients still recur within 5 years [5-7]. In the advanced setting, chemotherapy offers palliation and increases modestly the survival. However, the median overall survival (OS) is still approximately only 1 year after first-line and only 6 months after second-line treatment $[8,9]$. Intensive chemotherapy regimen recently failed to improve results $[10]$. The existence of molecular alterations in biliary tract cancers allows the development of targeted therapies [11]. However, the development of drugs is rendered difficult by the multiplicity of the targets in a population of patients with relatively rare tumors [12].

As for many types of cancers, targeting the tumor microenvironment (TME) offers another attractive avenue for drug development, which is particularly relevant in CCA. Indeed, CCA is recognized as a tumor with an important desmoplastic reaction, due to the stromal cells, and the infiltration of the tumor by different types of immune cells (Figure 2). Changes in the TME of CCA was also associated with prognosis in multiple studies [13]. Importantly, targeting the TME could also be conducted in combination with other treatment modalities, including cytotoxic chemotherapy and tumor cells targeted therapies, with opportunities of synergies.

In this review, we will describe the role of different compartments of the TME in CCA, focusing on the preclinical rationale for the development of new therapies. We will finally propose our opinion on how the development of innovative treatments targeting the TME could be conducted in CCA.

\section{TME is a clinically relevant histological hallmark of CCA}


Growing evidence demonstrates that TME contributes to cancer onset and progression in various cancers, including CCA [14-16]. The presence of a complex desmoplastic stroma is a distinctive histological feature of CCA (Figure 2A-B). TME consists of an intricate ecosystem made of non-tumor cells interacting with components of the extracellular matrix (ECM) and soluble factors (Figure 2C). A wide variety of cells populates the TME, including endothelial cells, pericytes, cancer-associated fibroblasts (CAFs) and immune cells [13]. The dynamic crosstalk between the TME and CCA tumor cells modulates cancer-associated processes, including cell death and proliferation, angiogenesis, invasion, metastasis and chemoresistance [17]. Soluble factors such as cytokines, chemokines, growth factors and extracellular vesicles play a key role in orchestrating these interactions $[14,18,19]$.

The clinical relevance of the stroma has been reported in several solid tumors, including breast cancer, colorectal cancer and CCA [20-22]. Importantly, these studies suggest that the stroma may exhibit a protective or an aggravating value depending on the cancer types. Thus, in breast cancer, patients with a stroma-high tumor have a worse prognosis compared to patients with a stroma-low tumor. In addition, the prognostic value of the tumor-stroma ratio is most discriminative in patients with grade III or triple-negative breast cancer [21]. In resectable colorectal cancer, an artificial intelligence algorithm based on a convolutional neural network model using transfer learning demonstrated that the tumor-stroma ratio is an independent predictor of prognosis, a high amount of stroma being associated with a reduced OS [22]. Similarly, a nationwide population-based study in Denmark reported that a high amount of stroma is an independent prognostic marker of an adverse outcome in patients with stage II 
colon cancer [23]. However, in iCCA, the stromal to tumor area ratio was inversely correlated with vascular invasion and positively correlated with a well-differentiated tumor grade. Actually, iCCA with more fibrous and less cellular features were associated with a better outcome. Patients with high stromal area exhibit a better disease-free survival (DFS), suggesting that desmoplastic stroma is protective in iCCA, possibly through a limitation of tumor invasiveness by building a barrier against tumor diffusion [20]. In addition, a high collagen reticulation index, defined as the number of collagen fiber branches within the entire length of the collagen network, was associated with a poorer OS [20]. Thus, not only the abundance of the stroma but also its reticulation and stiffness may influence the properties of the stroma and ultimately its prognostic value.

\section{Transcriptomic profiling highlights TME heterogeneity in CCA}

In 2013 , by combining laser capture microdissection and gene expression profiling, we highlighted transcriptomics alterations occurring in stromal cells of iCCA. A signature made of 1,073 genes was shown to discriminate the stromal compartment of iCCA tumors from the fibrous tissues in the adjacent non-tumor livers [24,25]. Notably, we demonstrated that osteopontin and TGF $\beta 2$ are both overexpressed in the stroma of iCCA. The expression of these two genes exhibited a heterogeneous expression and a high expression was demonstrated to correlate with a poor outcome, highlighting interesting therapeutic targets $[24,25]$. More recently, the same approach was used to pinpoint the functional features and diversity of stromal cells [26]. The study demonstrated that the TME of iCCA displays a large range of immunologic orientations and can be classified into four subtypes based on the cellular composition, including an 
immune desert subtype versus immunogenic, myeloid and mesenchymal subtypes. Importantly, this stromal classification is predictive of patient outcome [26]. Such immune-based classification might serve as a basis to design clinical trials evaluating specific targeted therapies, as we will develop in section 5.

These early transcriptomics studies mostly based on the microdissection of large fibrous areas highlighted the heterogeneity of the stroma in CCA and its complex transcriptional architecture. Deciphering the multifaceted nature of the stroma represents a great challenge, at both a basic and a translational level, to develop effective therapies. Addressing that particular issue, a recent study in iCCA took advantage of single-cell RNA-sequencing to unravel the complexity of the stroma at a cellular and molecular level $[27,28]$. This transcriptomic profiling at a single cell resolution highlighted a wide heterogeneity in the malignant, immune and stromal cells. Notably, the study identified 6 distinct fibroblast subsets. The predominant vascular-associated fibroblast subclass displayed a signature enriched for the microvasculature and showed a massive secretion of IL-6. Through functional analyses, the fibroblast-mediated secretion of IL-6 was shown to affect CCA cells malignancy through epigenetic mechanisms [28].

In summary, these transcriptomic studies allowed a better characterization of the alterations occurring in the TME of CCA, highlighting a high degree of transcriptional heterogeneity. They also provided a better insight into the interactions between tumors cells and TME, and they pave the way for the development of innovative therapeutic options targeting several components of TME.

\section{CAFs diversity offers various therapeutic orientations in CCA}




\subsection{CAFs are central protagonists in the TME of CCA}

Once the desmoplastic process is well engaged, the most predominant cells in the stroma are fibroblast-like cells referred to as cancer-associated fibroblasts (CAFs). CAFs form a heterogeneous group of cells characterized by the expression of phenotypic markers, including alpha-smooth muscle actin ( $\alpha-S M A)$ and platelet-derived growth factor receptor beta (PDGFRß) [29]. The origin of CAFs remains ambiguous in CCA. CAFs may derive from a wide variety of cells in the liver including portal fibroblasts, hepatic stellate cells (HSC) and bone marrow-derived mesenchymal precursor cells [16]. CAFs are first recruited by CCA tumor cells which subsequently sustain their activity. Once activated, CAFs promote CCA progression through the secretion of various soluble factors. For instance, transforming growth factor beta (TGF $\beta$ ), stromal cellderived factor 1 (SDF1), hepatocyte growth factor (HGF), connective tissue growth factor (CTGF) and epidermal growth factor (EGF) are extensively secreted by CAFs [30]. CAFs are central protagonists in the TME (Figure 3). They are involved in multidimensional interactions and influence the activity of a wide variety of cells including immune cells, lymphatic endothelial cells (LECs) and CCA tumor cells. For instance, Cadamuro and colleagues described a paracrine loop within the tumor reactive stroma of CCA, unleashed by PDGFD and involving CCA tumor cells, CAFs and LECs [29]. By secreting PDGFD, tumor cells induce the release of VEGFC and VEGFA by CAFs leading to the expansion of the lymphatic vasculature and tumor cell intravasation (Figure 3). This study highlights CAFs as potential targets in the TME for the treatment of CCA. Indeed, inducing CAFs apoptosis or targeting PDGFD-induced axis may 
represent an effective tool to block tumor-associated lymphangiogenesis and impede CCA tumor cell invasion [29].

CAFs are also involved in a reciprocal interplay with immune cells. Notably, CAFs expressing the fibroblast activation protein (FAP) are part of a specific fibroblast subset exhibiting a pro-inflammatory phenotype. Growing evidence show that FAP+CAFs could mediate tumor-promoting inflammatory and immunosuppressive effects (Figure 3). More specifically, FAP+ CAFs are able to foster the recruitment of myeloid-derived suppressor cells (MDSCs) in a STAT3 signaling-dependent manner [31,32].

\subsection{CAFs-oriented therapeutic strategies}

CAFs phenotype is clinically relevant for CCA treatment and represents an independent prognostic factor for OS [33]. Over the last decade, targeting CAFs has therefore been considered as a robust therapeutic strategy in CCA. For instance, by antagonizing PDGFRß and VEGFR3 (through imatinib mesylate), or by neutralizing VEFGA and VEGFC (respectively through SU5416 and SAR131675), the trans-LEC migration of CCA cells was alleviated. Moreover, a navitoclax-induced CAFs depletion triggers the reduction of the tumor-associated lymphangiogenesis in a rat model of CCA [29]. A combined administration of gemcitabine and nintedanib, an antifibrotic drug, was recently shown to reduce iCCA aggressiveness in mice through the reduction of prooncogenic soluble factors secreted by CAFs such as IL-6 and IL-8. Thus, the growth of iCCA xenografted tumors was shown to be significantly reduced [34]. Photothermal depletion of CAFs was also shown to normalize tumor stiffness in a mouse model of desmoplastic CCA [35]. We also recently reported that tumors developed from CCA cells resistant to EGFR inhibition in mice exhibited a more prominent stromal 
compartment enriched in CAFs [36]. CAFs were shown to contribute to chemoresistance of CCA cells by producing IGF2. Thus, the study provides a rationale for testing combined therapies against EGFR and IR/IGF1R in CCA [36].

Overall these recent studies contributed to better appreciate the diversity of CAFs and their respective functionalities in CCA, opening promising opportunities for clinical trials targeting CAFs (Figure 3).

\section{Targeting ECM in CCA}

The abundant presence of CAFs in the TME is also responsible for an aberrant production of ECM. CCA progression, dissemination and treatment are critically supported by ECM [37]. ECM is a highly reactive scaffold formed by a wide array of multifunctional molecules, encompassing collagens and non-collagenous glycoproteins, proteoglycans, glycosaminoglycans, and polysaccharides [37]. ECM remodeling is a key feature of CCA carcinogenesis, modulating signaling pathways involved in tumor progression. ECM is also a reservoir of soluble factors acting in the crosstalk between tumor cells and the stroma (Figure 2) [38]. Accordingly, the characterization of ECM composition and structure constitutes a promising line of investigation from a prognostic perspective [39]. As mentioned above, ECM proteins (e.g. osteopontin) are clinically relevant biomarkers associated with patient prognosis. In addition, growing evidence demonstrated that the overexpression of periostin (POSTN) and distinct mesothelin forms are able to predict a poor prognosis in a rat desmoplastic model of CCA [40]. Mechanistically, ECM proteins, including POSTN, tenascin-C and osteopontin were shown to modulate pro-tumorigenic functions by activating specific intracellular 
pathways, such as PI3K/AKT or MAPK, and thus promoting tumor cell proliferation, migration and invasion [37]. An exhaustive characterization of ECM at the protein level was very recently emphasized by Carpino and colleagues who reported proteome profiles specific of the TME in iCCA. This matrisome unraveled increased levels of fibrillar collagens and POSTN. The stiffness of the desmoplastic stroma was also explained by the near absence of proteoglycans and elastic fibers. A wide range of proteins involved in the maintenance of the basement membrane were also found downregulated [41]. Notably, evidence was provided that COL3A1 promotes iCCA cells migration as a component of tumor-associated aligned collagen, supporting COL3A1 as a promising therapeutic target to hamper tumor expansion and metastatic dissemination [41]. Interestingly, COL3A1 expression was reported as a prognostic biomarker in earlystage ovarian carcinoma patients, being associated with a shorter overall survival [42]. Similarly, COL3A1 overexpression was shown to confer a poor prognosis (reduced OS and DFS) in patients with bladder cancer [43]. In a mouse model of bleomycin-induced pulmonary fibrosis associated with ECM deposition, histone deacetylase inhibitors were shown to decrease COL3A1 expression, opening interesting therapeutic opportunities targeting ECM components [44].

Changes in the composition and the structure of ECM within TME can also negatively impact the response to therapies [45]. Indeed, both the density and the stiffness of the stroma can create an impenetrable barrier, concealing tumor cells from therapeutic agents. Steric hindrance also leads to an impaired supply in nutrients, oxygen and metabolites resulting in an increased hypoxia and metabolic stress, which could lead to the activation of anti-apoptotic and drug resistance pathways. In addition, 
modification of the ECM components have been reported to be involved in the response of tumor cells to radiotherapy [46]. Accordingly, addressing the TME in early drug discovery has been recommended as a strategy to overcome drug resistance and identify novel targets for cancer therapy [45].

In the light of the functional impact of ECM components on tumor progression and resistance to treatment, it comes natural to consider degrading this fibrous stroma as a therapeutic strategy to improve the care of patients with CCA. For instance, current therapies employed for CCA could be combined with a targeting of collagen or hyaluronan synthesis in order to improve their efficacy. The regulation of pathways upstream ECM synthesis could also represent an option. Notably, inhibition of the TGF $\beta$ signaling pathway to reduce ECM stiffness could be a relevant strategy. However, given that the abundance of the desmoplastic stroma has been recently associated with a better outcome in iCCA [20], it seems crucial to consider not only the amount of ECM deposition but also the composition, organization, and post-translational modifications of ECM. Accordingly, unsatisfactory results were obtained with pegylated recombinant human hyaluronidase combined with chemotherapy in patients with metastatic pancreatic cancer in a phase 1b/2 clinical trial [47].

\section{Targeting the immune microenvironment in $\mathrm{CCA}$}

The better understanding of the tumor immune microenvironment (TIME) led to the recent revolution of immunotherapy in oncology [48]. Mechanisms of the immune surveillance were described, and consequently also the mechanisms developed by tumors to escape this immune surveillance, focusing primarily on CD8+ T cells effectors 
[49-51]. The most widely immune checkpoints targeted to date for immunotherapy are programmed death-1 (PD-1) and its ligand PD-L1, as well as cytotoxic T-lymphocyteassociated protein 4 (CTLA-4), with numerous drugs targeting these pathways. Moreover, multiple other immune checkpoints are currently the focus of new drug development, and targeting of other effectors than CD8+ T cells is also studied. Here, we will describe the current knowledge about the role of TIME in CCA, starting from the type of cells then moving to functionality, in each case focusing on prognostic information related to the TIME.

\subsection{Description of the tumor immune microenvironment in CCA}

The study of all types of immune cells seems to be important to completely understand prognosis of CCA [52]. Regarding the adaptive immune system, lymphocytes are the main effectors of anti-tumor immune responses [53]. Tumor-infiltrating lymphocytes (TILs) have been described in CCA and are associated with the prognosis [54]. This is the case for both iCCA and eCCA [52,55]. Specifically, the presence of CD8+ lymphocytes seems to be associated with a better prognosis [51]. Regarding the innate immune system, Natural Killer (NK) cells are important components of tumor surveillance [56]. Epidemiological data suggest that polymorphisms of important genes related to NK cells functions (KIR and NKG2D) might be associated with the risk of developing CCA $[57,58]$, suggesting an important role of these cells on the immune surveillance against CCA. Furthermore, the infiltration by NK cells has been associated with better survival in resected iCCA [59]. Tumor-associated macrophages (TAMs) are important regulators of the tumoral immune response. In the liver, TAMs can be derived from Kupffer cells or from circulating bone-marrow derived macrophages. More 
importantly TAMs are divided between the M1 subtype, favoring anti-tumor immune response, and the M2 subtype, exerting immunosuppressive effects [60]. The presence of TAMs, and specifically of M2 TAMs has been associated with worse prognosis in CCA $[61,62]$. Moreover, CCA cells were shown in vitro to interact with macrophages, providing them with tumor-promoting capacities [63,64]. TAMs could also stimulate directly CCA tumor cells, notably through the Wnt/beta-catenin pathway [65]. Albeit usually considered of less importance, tumor-associated neutrophils could also play an important prognostic role in CCA [52].

\subsection{Functional description}

The importance of the immune infiltration in a subset of CCA could also be studied by gene expression analysis [66]. In a study of resected iCCA, Sia and colleagues described 2 classes of tumors, the proliferation and the inflammation classes. However, this analysis was done on the bulk of the tumors. As mentioned previously, a recent analysis focused on the stromal component, using laser microdissection [26]. The gene expression profile of the TME could then describe 4 TME subtypes. Subtype I1 displayed an immune desert, subtype 12 showed a reactive immunogenic pattern, subtype 13 displayed a myeloid-rich infiltrate, while subtype 14 was dominated by activated fibroblasts. These different subtypes were associated with clear differences in prognosis [26].

The functionality of the cells is of course of paramount importance, as we suggested when discussing the differences between M1 and M2 TAMs. One first evaluation could be done through the expression of immune checkpoints. Expression of PD-L1 was associated with infiltrations by TAMs and neutrophils [67]. There is conflicting evidence 
about the potential prognostic role of the expression of PD-L1 in CCA $[68,69]$. Technical issues and the selection of antibody might influence the results [70]. A meta-analysis suggested that PD-L1 expression was related to the tumor stage, which might explain discrepancies in results of various studies, and that overall PD-L1 expression was not associated with prognosis [71]. Another study suggested that PD-L1 expression depends on the etiology of iCCA [72]. Finally, the type of cells expressing PD-L1 might also influence the prognosis: TAMs expressing PD-L1 might be particularly important [73]. CTLA-4 expression might also have a prognostic role [74]. Expression on the adjacent tissue might be specifically important [75]. Finer evaluation of the functionality of the cells could be critical, using for example multiparametric flow cytometry or cytometry by time of flight (CyTof) but has rarely been performed in CCA. A study in eCCA using immunohistochemistry suggested that CD8+ or PD-L1+ cells were not associated with survival, but that focusing on CD8+CD54RO+ memory cells, a clear prognostic value was demonstrated [76]. In conclusion, more insight may be gained using refined techniques to characterize the functionality of the TIME in CCA.

\subsection{Consequences for drug development}

The role of the TIME in CCA, but mainly the efficacy of immune checkpoint inhibitors (ICl) in other tumor types, led to the development of clinical trials. Despite early disappointing results when $\mathrm{ICl}$ were used as monotherapy, phase 3 trials were launched and results are awaited. However, these trials did not use selection based on the immune contexture. In case of negativity, testing $\mathrm{ICl}$ in specific subgroups based on a biomarker might prove more interesting. 


\section{Targeting angiogenesis}

Targeting angiogenesis through VEGF inhibition showed modest results in many tumor types. As in most solid tumors, angiogenesis plays an important role in the development of CCA, and was showed to have prognostic value [77]. Regorafenib, a multitarget tyrosine kinase inhibitor targeting notably VEGFR2, was showed to have modest activity in CCA [78]. However, recent focus changed to using inhibition of VEGF in combination with other treatments. The concept of vascular normalization partially explains possible synergy between VEGF inhibition and improved penetration of other treatments inside the tumor [79]. Moreover, VEGF also plays a regulating role for immune cells, favoring by numerous ways the immunosuppressive components of the TIME, such as Tregs and M2 TAMs [80]. Finally, other angiogenic cytokines, such as TIE-2, also act in interplay between angiogenesis and immune infiltration [81]. It is thus probable that targeting angiogenesis in CCA will play a role only in combination with other treatment strategies.

\section{Past and ongoing clinical trials targeting TME in CCA}

The better characterization of different components of the TME has only recently lead to clinical trials. The development of immune-oncology lead to the trials of different antiPD-(L)1 inhibitors. However, results were overall disappointing when the drugs were used as monotherapy [82]. Nevertheless, recent results of combination with chemotherapy might prove more interesting [83]. Based on these results, different phase 3 trials were launched comparing standard chemotherapy to the combination of chemotherapy with anti-PD-(L)1 (clinicaltrials.gov identifiers NCT04003636, NCT03875235). The targeting of the TGF $\beta$ pathway is currently being tested in the 
clinics. Specifically, the TGF $\beta$ inhibitor galunisertib is currently under investigation for the treatment of metastatic pancreatic adenocarcinoma in combination with durvalumab (NCT02734160), and in different setting of hepatocellular carcinoma (as monotherapy NCT02240433 or in combination with sorafenib NCT02178358). However, no clinical trials were conducted in CCA. Finally, angiogenesis inhibition was tested in 2 trials using regorafenib, with interesting results $[78,84]$. Advantages and limitations of the current strategies targeting the TME in CCA are summarized in Table 1.

\section{Conclusion}

Studies performed over the last decade clearly highlighted the important role played by the TME in CCA, not only in tumor onset and progression, but also in the lack of efficacy of current treatments. Large-scale genomic approaches demonstrated the extreme heterogeneity of TME, both at the molecular and the cellular level. Clinically relevant subtypes of CCA were delineated based on TME specific features and relevant cell types and/or signaling pathways were identified for possible therapeutic targeting.

\section{Expert opinion}

Cytotoxic chemotherapy remains the mainstay of the medical treatment of CCA. However, its limitations are numerous, and do not appear to be easily overcome by novel drugs. Cytotoxic chemotherapy will probably continue to play a role in the foreseeable future, especially in patients with no targetable molecular alterations. In contrast, targeting molecular alterations appears very promising and will probably 
become available in the next few years [11]. However, such treatments frequently have other limitations, such as resistance mechanisms, and difficulty to penetrate the desmoplastic stroma of CCA. Also, they can only be applied in the subgroup of patients whose tumor harbors specific alterations and for which a drug is available. Limitations of these strategies centered on the tumor cells could be mitigated by combination with treatments targeting the TME. Indeed, targeting the CAFs could help other treatments to exert direct cytotoxic effects. In return, targeting the TME could only produce significant clinical efficacy if another treatment refrains the proliferation of tumor cells.

Different components of the TME also show complex interplays. The TIME interacts with endothelial cells and CAFs. Positive effects of combination of $\mathrm{ICl}$ and antiangiogenics drugs were already demonstrated in multiple tumor types. Such combination of treatment targeting different components of the TME might thus be a promising approach for developing new treatment strategies in CCA.

Combination strategies of different modalities might also be an important mean to avoid the emergence of resistance mechanisms. Selection pressure with a monotherapy usually leads in clinics to the development of a clone of tumor cells resistant to the treatment. The emergence of a resistant clone would be mitigated if a treatment targeting an alternative pathway is combined. The emergence of a resistance clone could also be less probable if normalization of the vascularization occurs following use of an antiangiogenics drug, enabling for stronger inhibition in the whole bulk of the tumor.

Another important parameter for the development of alternative treatment strategies will be the discovery of relevant biomarkers. As the screening for molecular alterations guide the treatment with targeted therapies, similar strategies could be applied with 
other treatment modalities. We have seen that there is inter-patients heterogeneity in the immune infiltrate of CCA; this is thus probable that a 'one-fit-all' approach will not lead to the best results. If we apply this reasoning to the subtype of TME described by Job and colleagues, we might propose that the II subtype of tumors with an immune desert should receive a treatment favoring immune infiltration; subtype 12 showing a reactive immunogenic pattern could best be treated by $\mathrm{ICl}$; subtype $\mathrm{I} 3$ displaying a myeloid-rich infiltrate might be best treated with $\mathrm{ICl}$-antiangiogenics combination; finally the subtype 14 dominated by activated fibroblasts should be treated with drugs inhibiting fibroblasts activation [26]. Thus, a rationale drug development based on building adequate biomarkers for each drug is clearly an important prerequisite to success of targeting the TME.

Additional significant difficulty in the development of these strategies is the multiple actors involved (different pharmaceutical companies developing drugs; different academicals groups focusing on different treatment strategies), sometimes competing for developing treatments at the same time for the same patients population. While the development of a biomarker might be the best option when focusing on patient's benefit, short-term development of a 'one-fit-all' approach is frequently easier to conduct. Coordination of different actors in a strategy trying to combine targeting of different pathways is also an important challenge. Finally, an important difficulty of the combinations is also the risk for increased, sometimes synergistic toxicities precluding effective application in the clinical setting.

Thus, we believe that the development of treatment targeting the TME will produce the best results if thought as a combination with cytotoxic treatments (chemotherapy 
and/or targeted therapies depending on the molecular alterations presented by the tumor), as well as a combination of treatments targeting different components of the TME (Figure 4). Positive results would also be clearer if biomarkers are developed to define the population of interest for each combination strategy.

\section{Funding}

This work was supported by Inserm, Université de Rennes 1, and ITMO Cancer AVIESAN (Alliance Nationale pour les Sciences de la Vie et de la Santé) dans le cadre du Plan cancer (Non-coding RNA in cancerology: fundamental to translational), Ligue Contre le Cancer (CD35, CD22, CD49, CD85). CL is supported by a PhD fellowship from Université de Rennes 1. CC and JE are members of the European Network for the Study of Cholangiocarcinoma (ENSCCA) and participate in the initiative COST Action EURO-CHOLANGIO-NET granted by the COST Association (CA18122).

\section{Declaration of Interest}

$\mathrm{CL}$ and $\mathrm{CC}$ have no relevant affiliations or financial involvement with any organization or entity with a financial interest in or financial conflict with the subject matter or materials discussed in the manuscript. This includes employment, consultancies, honoraria, stock ownership or options, expert testimony, grants or patents received or pending, or royalties. JE is expert and receives honoraria from MSD, Roche, AstraZeneca and BMS.

\section{Figure legends}


Figure 1. Anatomical classification of CCA malignancies along the biliary tract.

Figure 2. Overview of the tumor microenvironment (TME) in CCA. (A) Hematoxylin and Eosin staining of a representative human CCA tumor tissue. (B) Sirius red staining of a representative human CCA tumor tissue. Both staining are highlighting the abundant stroma in CCA. (C) Schematic overview of the intricate ecosystem constitutive of the TME. This environment is populated with a wide variety of cells including CCA cancer cells, cancer-associated fibroblasts (CAFs), myeloid-derived suppressor cells (MDSCs), tumor-associated macrophages (TAMs), tumor-infiltrating lymphocytes (TILs), tumorassociated neutrophils (TANs) and natural killer cells (NKs). These cells interact together as well as with components of the extracellular matrix (ECM) and soluble factors within the TME to modulate CCA onset and progression.

Figure 3. Examples of mechanisms by which the tumor microenvironment fosters CCA onset and progression. (A) Interactions of cancer-associated fibroblasts (CAFs) and/or tumor-associated macrophages (TAMs) with CCA tumor cells promote the secretion of various soluble factors to induce extracellular matrix (ECM) remodeling and angiogenesis. (B) By secreting platelet-derived growth factor D (PDGFD), tumor cells induce the release of vascular endothelial growth factor A (VEGFA) and C (VEGFC) by CAFs leading to the expansion of the lymphatic vasculature and tumor cell intravasation. (C) Mechanisms developed by tumors to escape immune surveillance. Notably, fibroblast activation protein positive $(\mathrm{FAP}+)$ CAFs mediate tumor-promoting inflammatory and immunosuppressive effects through the recruitment of myeloid-derived suppressor cells (MDSCs) in a STAT3 signaling-dependent manner. 
Immunosuppressive effects of CAFs on tumor-infiltrating lymphocytes (TILs), tumorassociated neutrophils (TANs) and natural killer cells (NKs) are shown.

Figure 4. Outlook on the therapies targeting the CCA tumor microenvironment in combination with cytotoxic chemotherapy.

\section{References}

Papers of special note have been highlighted as either of interest $(\bullet)$ or of considerable interest $(\bullet)$ to readers.

1. Banales JM, Marin JJG, Lamarca A, et al. Cholangiocarcinoma 2020: the next horizon in mechanisms and management. Nat Rev Gastroenterol Hepatol. 2020 Sep;17(9):557-588.

- This paper is of considerable interest by providing a recent state-of-the-art consensus statement from the ENSCCA network concerning the next horizon in the management of CCA.

2. Louis C, Papoutsoglou P, Coulouarn C. Molecular classification of cholangiocarcinoma. Curr Opin Gastroenterol. 2020 Mar;36(2):57-62.

3. Saha SK, Zhu AX, Fuchs CS, et al. Forty-Year Trends in Cholangiocarcinoma Incidence in the U.S.: Intrahepatic Disease on the Rise. Oncologist. 2016 May;21(5):594-9. 
4. Bridgewater J, Galle PR, Khan SA, et al. Guidelines for the diagnosis and management of intrahepatic cholangiocarcinoma. J Hepatol. 2014 Jun;60(6):1268-89.

5. Lamarca A, Edeline J, McNamara MG, et al. Current standards and future perspectives in adjuvant treatment for biliary tract cancers. Cancer Treat Rev. 2020 Mar;84:101936.

6. Malka D, Edeline J. Adjuvant capecitabine in biliary tract cancer: a standard option? Lancet Oncol. 2019 May;20(5):606-608.

7. Primrose J, Falk S, Finch-Jones M, et al. Systemic chemotherapy with or without cetuximab in patients with resectable colorectal liver metastasis: the New EPOC randomised controlled trial. Lancet Oncol. 2014 May;15(6):601-11.

8. Rizzo A, Ricci AD, Tober N, et al. Second-line Treatment in Advanced Biliary Tract Cancer: Today and Tomorrow. Anticancer Res. 2020 Jun;40(6):3013-3030.

9. Valle J, Wasan $\mathrm{H}$, Palmer $\mathrm{DH}$, et al. Cisplatin plus gemcitabine versus gemcitabine for biliary tract cancer. N Engl J Med. 2010 Apr 8;362(14):1273-81.

10. Phelip JM, Edeline J, Blanc JF, et al. Modified FOLFIRINOX versus CisGem firstline chemotherapy for locally advanced non resectable or metastatic biliary tract cancer (AMEBICA)-PRODIGE 38: Study protocol for a randomized controlled multicenter phase II/III study. Dig Liver Dis. 2019 Feb;51(2):318-320.

11. Lamarca A, Barriuso J, McNamara MG, et al. Molecular targeted therapies: Ready for "prime time" in biliary tract cancer. J Hepatol. 2020 Jul;73(1):170-185.

12. Verlingue $L$, Hollebecque $A$, Boige $V$, et al. Matching genomic molecular aberrations with molecular targeted agents: Are biliary tract cancers an ideal playground? Eur J Cancer. 2017 Aug;81:161-173. 
- This paper is of interest by clearly reviewing the potential for precision medicine in biliary tract cancers.

13. Fabris L, Sato K, Alpini G, et al. The Tumor Microenvironment in Cholangiocarcinoma Progression. Hepatology. 2020 Jun 4.

- This paper is of interest by recently reviewing in a comprehensive manner the role of microenvirenment in CCA progression.

14. Fabris L, Perugorria MJ, Mertens J, et al. The tumour microenvironment and immune milieu of cholangiocarcinoma. Liver Int. 2019 May;39 Suppl 1:63-78.

15. Paillet J, Kroemer G, Pol JG. Immune contexture of cholangiocarcinoma. Curr Opin Gastroenterol. 2020 Mar;36(2):70-76.

16. Vaquero J, Aoudjehane L, Fouassier L. Cancer-associated fibroblasts in cholangiocarcinoma. Curr Opin Gastroenterol. 2020 Mar;36(2):63-69.

17. Hanahan D, Coussens LM. Accessories to the crime: functions of cells recruited to the tumor microenvironment. Cancer Cell. 2012 Mar 20;21(3):309-22.

18. Lapitz A, Arbelaiz A, Olaizola P, et al. Extracellular Vesicles in Hepatobiliary Malignancies. Front Immunol. 2018;9:2270.

19. Hogdall D, Lewinska M, Andersen JB. Desmoplastic Tumor Microenvironment and Immunotherapy in Cholangiocarcinoma. Trends Cancer. 2018 Mar;4(3):239255.

20. Guedj N, Blaise L, Cauchy F, et al. Prognostic value of desmoplastic stroma in intrahepatic cholangiocarcinoma. Mod Pathol. 2020 Aug 28.

- This paper is of interest by reporting that a high abundance of the stroma is associated with a better outcome in CCA. 
21. Vangangelt $\mathrm{KMH}$, Green AR, Heemskerk IMF, et al. The prognostic value of the tumor-stroma ratio is most discriminative in patients with grade III or triplenegative breast cancer. Int J Cancer. 2020 Apr 15;146(8):2296-2304.

22. Zhao K, Li Z, Yao S, et al. Artificial intelligence quantified tumour-stroma ratio is an independent predictor for overall survival in resectable colorectal cancer. EBioMedicine. 2020 Nov;61:103054.

23. Eriksen AC, Sorensen FB, Lindebjerg J, et al. The prognostic value of tumour stroma ratio and tumour budding in stage II colon cancer. A nationwide population-based study. Int J Colorectal Dis. 2018 Aug;33(8):1115-1124.

24. Sulpice L, Desille M, Turlin B, et al. Gene expression profiling of the tumor microenvironment in human intrahepatic cholangiocarcinoma. Genom Data. 2016 Mar;7:229-32.

25. Sulpice L, Rayar M, Desille M, et al. Molecular profiling of stroma identifies osteopontin as an independent predictor of poor prognosis in intrahepatic cholangiocarcinoma. Hepatology. 2013 Dec;58(6):1992-2000.

- This study is of interest by reporting one of the first clinically relevant molecular profiles of the stroma in CCA.

26. Job S, Rapoud D, Dos Santos A, et al. Identification of four immune subtypes characterized by distinct composition and functions of tumor microenvironment in intrahepatic cholangiocarcinoma. Hepatology. 2019 Dec 25.

-• This paper is of considerable interest as it describes different subtypes of iCC and could have direct implication for the selection of patients for immune checkpoint inhibitors treatment. 
27. Fabris L, Andersen JB, Fouassier L. Intrahepatic cholangiocarcinoma: A singlecell resolution unraveling the complexity of the tumor microenvironment. $\mathrm{J}$ Hepatol. 2020 Nov;73(5):1007-1009.

28. Zhang M, Yang $\mathrm{H}$, Wan L, et al. Single-cell transcriptomic architecture and intercellular crosstalk of human intrahepatic cholangiocarcinoma. J Hepatol. 2020 Nov;73(5):1118-1130.

-• This study is of considerable interest. By considering the CCA stroma at a cellular level, this study paves the way to unravel the complexity of CCA microenvironment.

29. Cadamuro M, Brivio S, Mertens J, et al. Platelet-derived growth factor-D enables liver myofibroblasts to promote tumor lymphangiogenesis in cholangiocarcinoma. J Hepatol. 2019 Apr;70(4):700-709.

30. Gentilini A, Pastore M, Marra F, et al. The Role of Stroma in Cholangiocarcinoma: The Intriguing Interplay between Fibroblastic Component, Immune Cell Subsets and Tumor Epithelium. Int J Mol Sci. 2018 Sep 22;19(10).

31. Lin $\mathrm{Y}, \mathrm{Li} \mathrm{B}$, Yang $\mathrm{X}$, et al. Fibroblastic FAP promotes intrahepatic cholangiocarcinoma growth via MDSCs recruitment. Neoplasia. 2019 Dec;21(12):1133-1142.

32. Yang $X$, Lin $Y$, Shi $Y$, et al. FAP Promotes Immunosuppression by CancerAssociated Fibroblasts in the Tumor Microenvironment via STAT3-CCL2 Signaling. Cancer Res. 2016 Jul 15;76(14):4124-35.

33. Zhang XF, Dong M, Pan YH, et al. Expression pattern of cancer-associated fibroblast and its clinical relevance in intrahepatic cholangiocarcinoma. Hum Pathol. 2017 Jul;65:92-100. 
34. Yamanaka T, Harimoto N, Yokobori T, et al. Nintedanib inhibits intrahepatic cholangiocarcinoma aggressiveness via suppression of cytokines extracted from activated cancer-associated fibroblasts. Br J Cancer. 2020 Mar;122(7):986-994.

35. Nicolas-Boluda A, Vaquero J, Laurent G, et al. Photothermal Depletion of CancerAssociated Fibroblasts Normalizes Tumor Stiffness in Desmoplastic Cholangiocarcinoma. ACS Nano. 2020 May 26;14(5):5738-5753.

36. Vaquero J, Lobe C, Tahraoui S, et al. The IGF2/IR/IGF1R Pathway in Tumor Cells and Myofibroblasts Mediates Resistance to EGFR Inhibition in Cholangiocarcinoma. Clin Cancer Res. 2018 Sep 1;24(17):4282-4296.

37. Fabris L, Cadamuro M, Cagnin S, et al. Liver Matrix in Benign and Malignant Biliary Tract Disease. Semin Liver Dis. 2020 Aug;40(3):282-297.

38. Lorusso G, Ruegg C, Kuonen F. Targeting the Extra-Cellular Matrix-Tumor Cell Crosstalk for Anti-Cancer Therapy: Emerging Alternatives to Integrin Inhibitors. Front Oncol. 2020;10:1231.

39. Theocharis AD, Skandalis SS, Gialeli C, et al. Extracellular matrix structure. Adv Drug Deliv Rev. 2016 Feb 1;97:4-27.

40. Manzanares MA, Campbell DJW, Maldonado GT, et al. Overexpression of periostin and distinct mesothelin forms predict malignant progression in a rat cholangiocarcinoma model. Hepatol Commun. 2018 Feb;2(2):155-172.

41. Carpino G, Overi D, Melandro F, et al. Matrisome analysis of intrahepatic cholangiocarcinoma unveils a peculiar cancer-associated extracellular matrix structure. Clin Proteomics. 2019;16:37. 
42. Engqvist $\mathrm{H}$, Parris TZ, Kovacs $\mathrm{A}$, et al. Immunohistochemical validation of COL3A1, GPR158 and PITHD1 as prognostic biomarkers in early-stage ovarian carcinomas. BMC Cancer. 2019 Sep 18;19(1):928.

43. Yuan L, Shu B, Chen L, et al. Overexpression of COL3A1 confers a poor prognosis in human bladder cancer identified by co-expression analysis. Oncotarget. 2017 Sep 19;8(41):70508-70520.

44. Zhang X, Liu H, Hock $\mathrm{T}$, et al. Histone deacetylase inhibition downregulates collagen 3A1 in fibrotic lung fibroblasts. Int J Mol Sci. 2013 Sep 27;14(10):1960517.

45. Kaemmerer E, Loessner D, Avery VM. Addressing the tumour microenvironment in early drug discovery: a strategy to overcome drug resistance and identify novel targets for cancer therapy. Drug Discov Today. 2020 Dec 2.

46. Henke E, Nandigama R, Ergun S. Extracellular Matrix in the Tumor Microenvironment and Its Impact on Cancer Therapy. Front Mol Biosci. 2019;6:160.

47. Ramanathan RK, McDonough SL, Philip PA, et al. Phase IB/II Randomized Study of FOLFIRINOX Plus Pegylated Recombinant Human Hyaluronidase Versus FOLFIRINOX Alone in Patients With Metastatic Pancreatic Adenocarcinoma: SWOG S1313. J Clin Oncol. 2019 May 1;37(13):1062-1069.

- This paper is of interest. This phase 2 trial made the proof of concept of the targeting of tumor stroma. However, results of the drug were disappointing in phase 3. 
48. Binnewies M, Roberts EW, Kersten $\mathrm{K}$, et al. Understanding the tumor immune microenvironment (TIME) for effective therapy. Nat Med. 2018 May;24(5):541550.

49. Chen DS, Mellman I. Oncology meets immunology: the cancer-immunity cycle. Immunity. 2013 Jul 25;39(1):1-10.

50. Waldman AD, Fritz JM, Lenardo MJ. A guide to cancer immunotherapy: from T cell basic science to clinical practice. Nat Rev Immunol. 2020 Nov;20(11):651668.

51. Asahi $\mathrm{Y}$, Hatanaka KC, Hatanaka $\mathrm{Y}$, et al. Prognostic impact of CD8+ $\mathrm{T}$ cell distribution and its association with the HLA class I expression in intrahepatic cholangiocarcinoma. Surg Today. 2020 Aug;50(8):931-940.

52. Kitano Y, Okabe H, Yamashita YI, et al. Tumour-infiltrating inflammatory and immune cells in patients with extrahepatic cholangiocarcinoma. $\mathrm{Br} \mathrm{J}$ Cancer. 2018 Jan;118(2):171-180.

53. Badalamenti G, Fanale D, Incorvaia L, et al. Role of tumor-infiltrating lymphocytes in patients with solid tumors: Can a drop dig a stone? Cell Immunol. 2019 Sep;343:103753.

54. Goeppert B, Frauenschuh L, Zucknick M, et al. Prognostic impact of tumourinfiltrating immune cells on biliary tract cancer. Br J Cancer. 2013 Nov 12;109(10):2665-74.

55. Vigano L, Soldani C, Franceschini B, et al. Tumor-Infiltrating Lymphocytes and Macrophages in Intrahepatic Cholangiocellular Carcinoma. Impact on Prognosis after Complete Surgery. J Gastrointest Surg. 2019 Nov;23(11):2216-2224. 
56. Chiossone L, Dumas PY, Vienne M, et al. Natural killer cells and other innate lymphoid cells in cancer. Nat Rev Immunol. 2018 Nov;18(11):671-688.

57. Cornillet M, Jansson H, Schaffer M, et al. Imbalance of Genes Encoding Natural Killer Immunoglobulin-Like Receptors and Human Leukocyte Antigen in Patients With Biliary Cancer. Gastroenterology. 2019 Oct;157(4):1067-1080 e9.

58. Wadsworth CA, Dixon PH, Taylor-Robinson S, et al. Polymorphisms in Natural Killer Cell Receptor Protein 2D (NKG2D) as a Risk Factor for Cholangiocarcinoma. J Clin Exp Hepatol. 2019 Mar-Apr;9(2):171-175.

59. Fukuda $\mathrm{Y}$, Asaoka $\mathrm{T}$, Eguchi $\mathrm{H}$, et al. Endogenous $\mathrm{CXCL9}$ affects prognosis by regulating tumor-infiltrating natural killer cells in intrahepatic cholangiocarcinoma. Cancer Sci. 2020 Feb;111(2):323-333.

60. Noy R, Pollard JW. Tumor-associated macrophages: from mechanisms to therapy. Immunity. 2014 Jul 17;41(1):49-61.

61. Subimerb C, Pinlaor S, Khuntikeo N, et al. Tissue invasive macrophage density is correlated with prognosis in cholangiocarcinoma. Mol Med Rep. 2010 JulAug;3(4):597-605.

62. Thanee M, Loilome W, Techasen A, et al. Quantitative changes in tumorassociated M2 macrophages characterize cholangiocarcinoma and their association with metastasis. Asian Pac J Cancer Prev. 2015;16(7):3043-50.

63. Raggi C, Correnti M, Sica A, et al. Cholangiocarcinoma stem-like subset shapes tumor-initiating niche by educating associated macrophages. J Hepatol. 2017 Jan;66(1):102-115. 
64. Zeng J, Liu Z, Sun S, et al. Tumor-associated macrophages recruited by periostin in intrahepatic cholangiocarcinoma stem cells. Oncol Lett. 2018 Jun;15(6):86818686.

65. Loilome W, Bungkanjana P, Techasen A, et al. Activated macrophages promote Wnt/beta-catenin signaling in cholangiocarcinoma cells. Tumour Biol. 2014 Jun;35(6):5357-67.

66. Sia D, Hoshida Y, Villanueva A, et al. Integrative molecular analysis of intrahepatic cholangiocarcinoma reveals 2 classes that have different outcomes. Gastroenterology. 2013 Apr;144(4):829-40.

67. Kitano Y, Yamashita YI, Nakao Y, et al. Clinical Significance of PD-L1 Expression in Both Cancer and Stroma Cells of Cholangiocarcinoma Patients. Ann Surg Oncol. 2020 Feb;27(2):599-607.

68. Fontugne J, Augustin J, Pujals A, et al. PD-L1 expression in perihilar and intrahepatic cholangiocarcinoma. Oncotarget. 2017 Apr 11;8(15):24644-24651.

69. Walter D, Herrmann E, Schnitzbauer AA, et al. PD-L1 expression in extrahepatic cholangiocarcinoma. Histopathology. 2017 Sep;71(3):383-392.

70. Kriegsmann M, Roessler S, Kriegsmann K, et al. Programmed cell death ligand 1 (PD-L1, CD274) in cholangiocarcinoma - correlation with clinicopathological data and comparison of antibodies. BMC Cancer. 2019 Jan 15;19(1):72.

71. Xu G, Sun L, Li Y, et al. The Clinicopathological and Prognostic Value of PD-L1 Expression in Cholangiocarcinoma: A Meta-Analysis. Front Oncol. 2019;9:897.

72. Lu JC, Zeng HY, Sun QM, et al. Distinct PD-L1/PD1 Profiles and Clinical Implications in Intrahepatic Cholangiocarcinoma Patients with Different Risk Factors. Theranostics. 2019;9(16):4678-4687. 
73. Loeuillard E, Yang J, Buckarma E, et al. Targeting tumor-associated macrophages and granulocytic myeloid-derived suppressor cells augments PD-1 blockade in cholangiocarcinoma. J Clin Invest. 2020 Oct 1;130(10):5380-5396.

-• This study is of considerable interest by highlighting the potential of combining $\mathrm{ICl}$ therapies with immunotherapies targeting the microenvironment of CCA.

74. Lim YJ, Koh J, Kim K, et al. Clinical Implications of Cytotoxic T Lymphocyte Antigen-4 Expression on Tumor Cells and Tumor-Infiltrating Lymphocytes in Extrahepatic Bile Duct Cancer Patients Undergoing Surgery Plus Adjuvant Chemoradiotherapy. Target Oncol. 2017 Apr;12(2):211-218.

75. Ghidini M, Cascione L, Carotenuto $\mathrm{P}$, et al. Characterisation of the immunerelated transcriptome in resected biliary tract cancers. Eur J Cancer. 2017 Nov;86:158-165.

76. Kim R, Coppola D, Wang E, et al. Prognostic value of CD8CD45RO tumor infiltrating lymphocytes in patients with extrahepatic cholangiocarcinoma. Oncotarget. 2018 May 4;9(34):23366-23372.

77. Thelen A, Scholz A, Weichert W, et al. Tumor-associated angiogenesis and lymphangiogenesis correlate with progression of intrahepatic cholangiocarcinoma. Am J Gastroenterol. 2010 May;105(5):1123-32.

78. Sun W, Patel A, Normolle D, et al. A phase 2 trial of regorafenib as a single agent in patients with chemotherapy-refractory, advanced, and metastatic biliary tract adenocarcinoma. Cancer. 2019 Mar 15;125(6):902-909.

- This paper is of interest by suggesting the potential role of antiangiogenics in biliary tract cancers.

79. Viallard C, Larrivee B. Tumor angiogenesis and vascular normalization: alternative therapeutic targets. Angiogenesis. 2017 Nov;20(4):409-426. 
80. Fukumura D, Kloepper J, Amoozgar Z, et al. Enhancing cancer immunotherapy using antiangiogenics: opportunities and challenges. Nat Rev Clin Oncol. 2018 May;15(5):325-340.

- This review is of interest by emphasizing the association of antiangiogenic drugs with $\mathrm{ICl}$ therapies to improve patient outcomes.

81. Atanasov G, Dietel C, Feldbrugge L, et al. Angiogenic miRNAs, the angiopoietin axis and related TIE2-expressing monocytes affect outcomes in cholangiocarcinoma. Oncotarget. 2018 Jul 6;9(52):29921-29933.

82. Rizzo A, Ricci AD, Brandi G. Recent advances of immunotherapy for biliary tract cancer. Expert Rev Gastroenterol Hepatol. 2020 Nov 20.

83. Ueno M, Ikeda M, Morizane C, et al. Nivolumab alone or in combination with cisplatin plus gemcitabine in Japanese patients with unresectable or recurrent biliary tract cancer: a non-randomised, multicentre, open-label, phase 1 study. Lancet Gastroenterol Hepatol. 2019 Aug;4(8):611-621.

84. Demols A, Borbath I, Van den Eynde M, et al. Regorafenib after failure of gemcitabine and platinum-based chemotherapy for locally advanced/metastatic biliary tumors: REACHIN, a randomized, double-blind, phase II trial. Ann Oncol. 2020 Sep;31(9):1169-1177. 
Table 1: Current strategies used for targeting the TME in CCA.

\begin{tabular}{|c|c|c|}
\hline Strategies & Advantages & Limitations \\
\hline $\begin{array}{l}\text { Anti-PD-(L)1 } \\
\text { monotherapies }\end{array}$ & $\begin{array}{l}\text { Validated in many tumor } \\
\text { types } \\
\text { Easily available } \\
\text { Low toxicity }\end{array}$ & $\begin{array}{l}\text { Low efficacy } \\
\text { No predictive factors of } \\
\text { response } \\
\text { High costs }\end{array}$ \\
\hline $\begin{array}{l}\text { Anti-PD-(L)1 - } \\
\text { chemotherapy } \\
\text { combinations }\end{array}$ & $\begin{array}{l}\text { Validated in many tumor } \\
\text { types } \\
\text { Easily available } \\
\text { Acceptable toxicity }\end{array}$ & $\begin{array}{l}\text { No predictive factors of } \\
\text { response } \\
\text { High costs }\end{array}$ \\
\hline TGF $\beta$ inhibition & Low toxicity & $\begin{array}{l}\text { No predictive factors } \\
\text { Efficacy in combination } \\
\text { with anti-PD-(L)1 inhibitors } \\
\text { understudied }\end{array}$ \\
\hline Angiogenesis inhibition & $\begin{array}{l}\text { Certain level of efficacy } \\
\text { Toxicity of Regorafenib }\end{array}$ & $\begin{array}{l}\text { Potential synergy in } \\
\text { combination with anti-PD- } \\
\text { (L)1 inhibitors }\end{array}$ \\
\hline
\end{tabular}




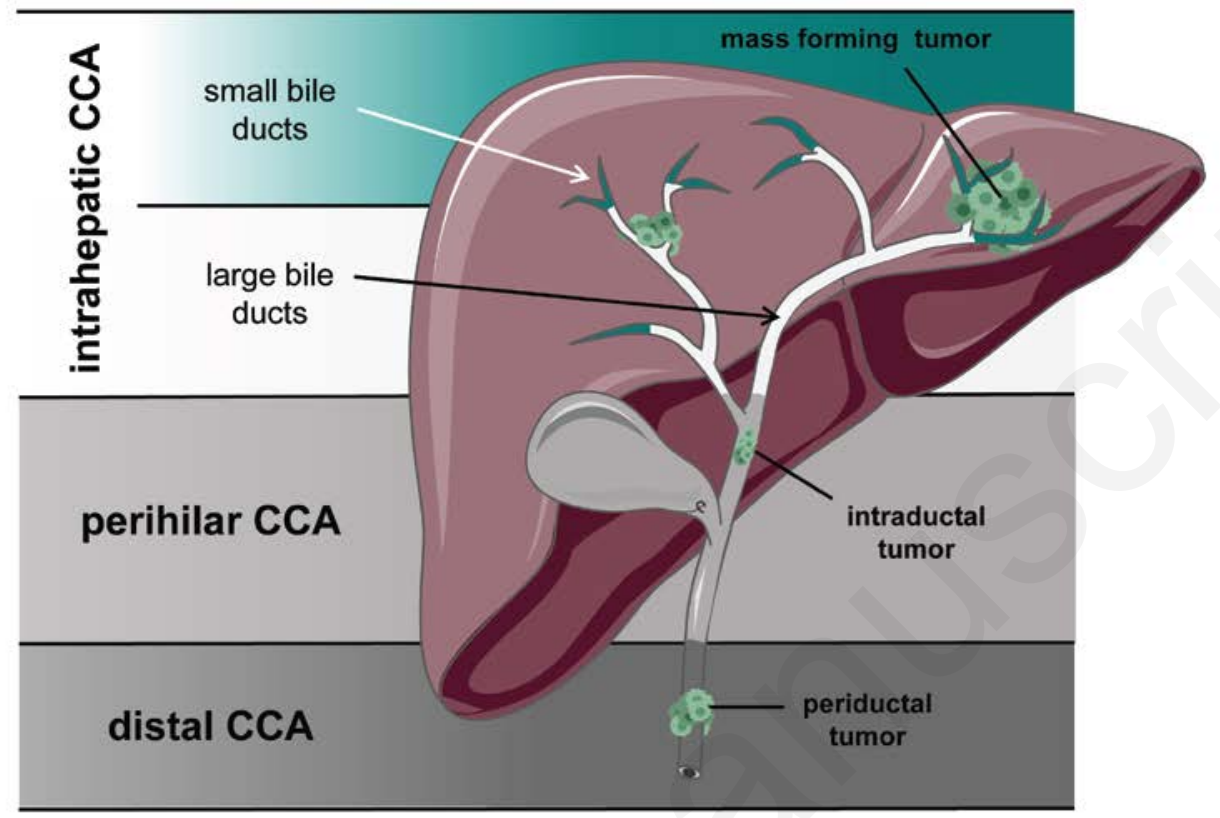

FIGURE 1 

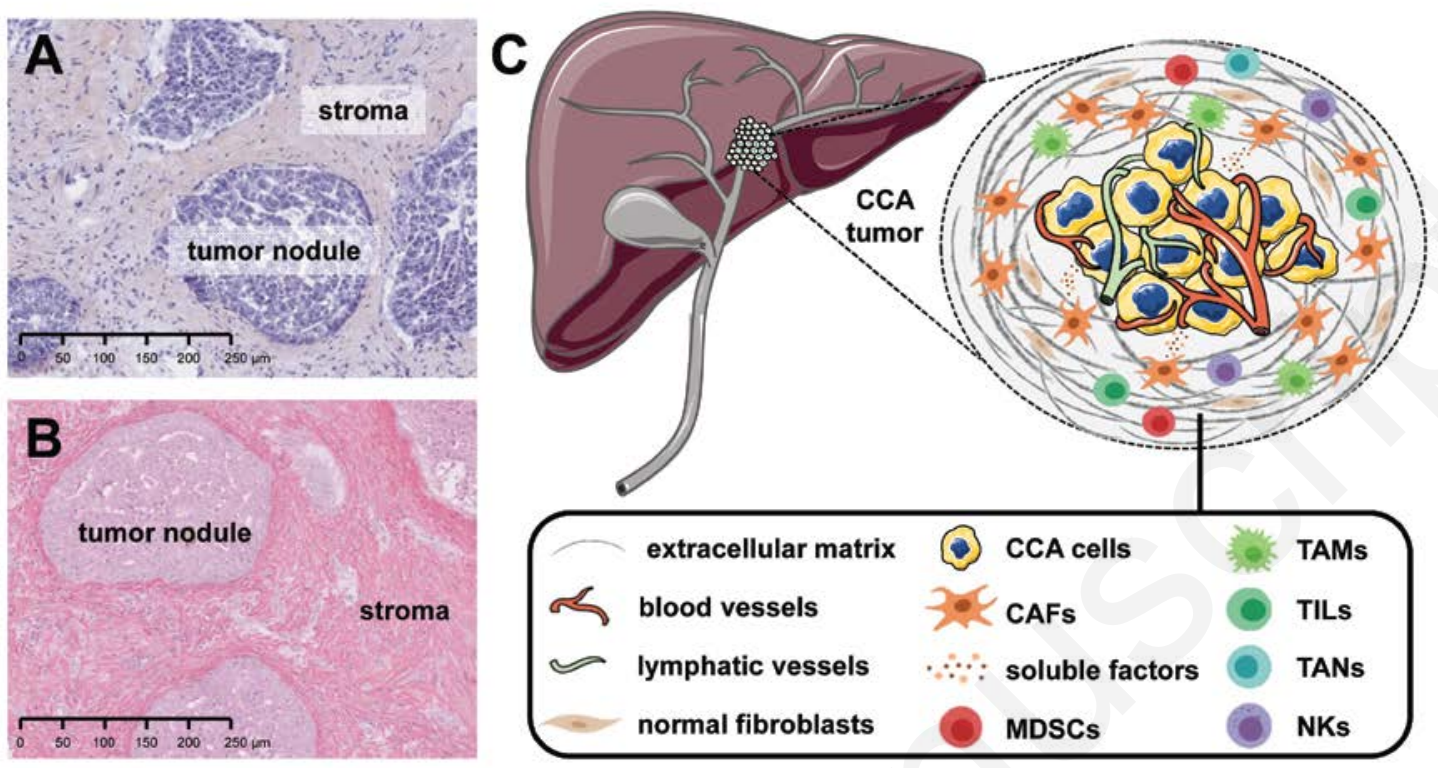

FIGURE 2 


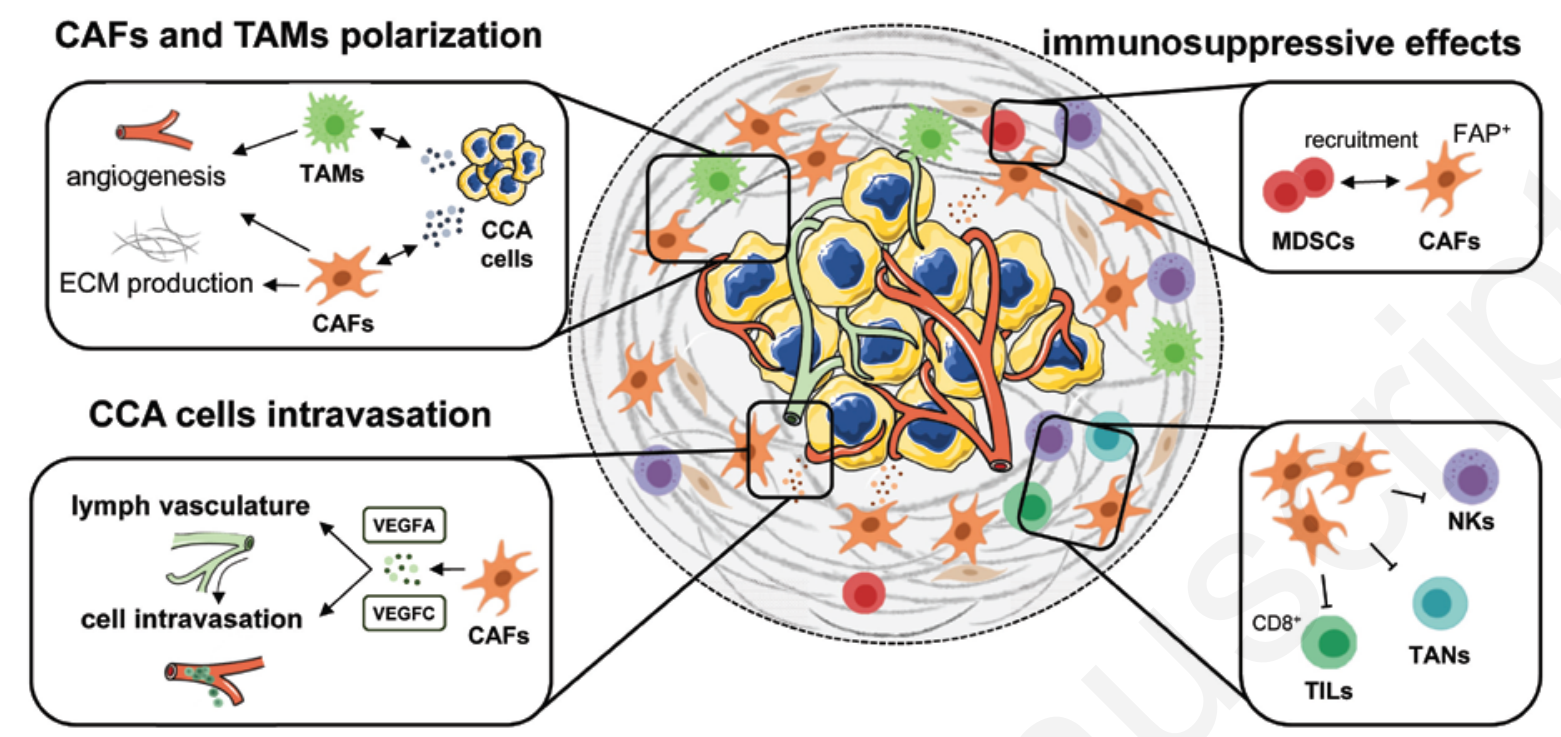

\section{FIGURE 3}




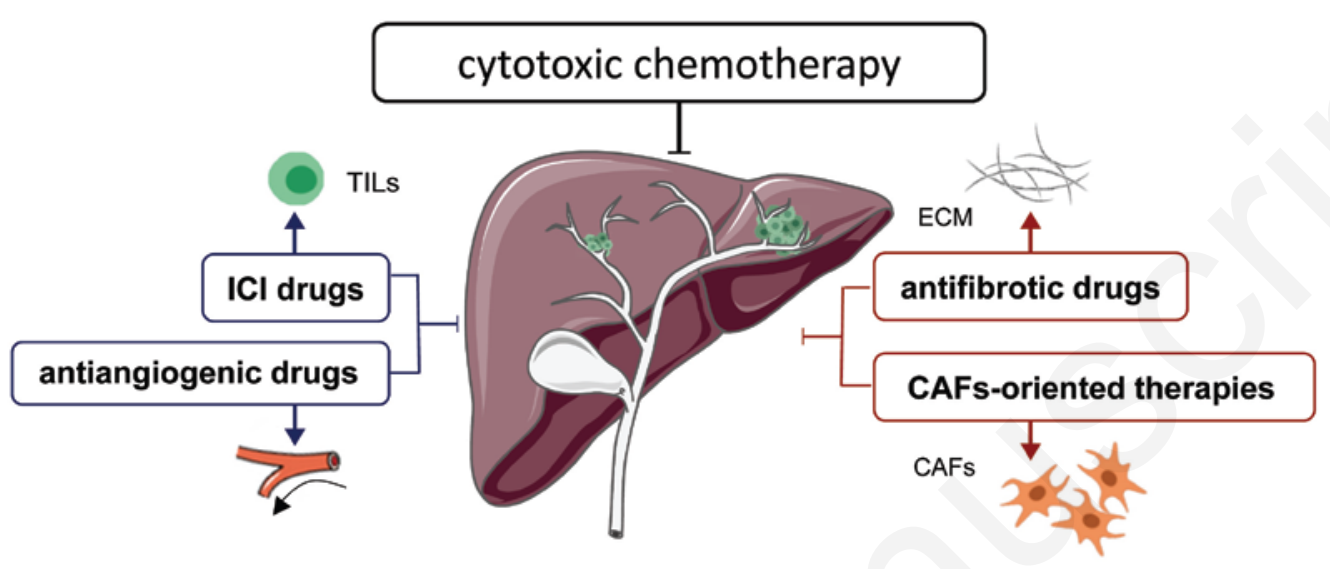

FIGURE 4 